\title{
Shear instabilities in the dust layer of the solar nebula II. Different unperturbed states
}

\author{
Minoru Sekiya ${ }^{1}$ and Naoki Ishitsu ${ }^{2}$ \\ ${ }^{1}$ Department of Earth and Planetary Sciences, Faculty of Sciences, 33 Kyushu University, Hakozaki, Fukuoka 812-8581, Japan \\ ${ }^{2}$ Department of Earth and Planetary Sciences, Graduate School of Sciences, 33 Kyushu University, Hakozaki, Fukuoka 812-8581, Japan
}

(Received December 11, 2000; Revised March 30, 2001; Accepted April 1, 2001)

\begin{abstract}
In the previous paper (Sekiya and Ishitsu, 2000), the shear instability in the dust layer of the solar nebula was investigated by using the constant Richardson number solution (Sekiya, 1998) as the unperturbed state, and the growth rate of the most unstable mode was calculated to be much less than the Keplerian angular velocity as long as the Richardson number was larger than 0.1. In this paper, we calculate the growth rate using different unperturbed states: (1) a sinusoidal density distribution, and (2) a constant density around the midplane with sinusoidal transition regions. An unperturbed state of this paper is considered to correspond to the first stage of shear instability that occurs as a result of dust settling in a laminar phase of the solar nebula. On the other hand, the unperturbed state of the previous paper corresponds to the quasi-equilibrium state (Sekiya, 1998) which is attained by the turbulent mixing. The results show that the growth rate is much larger than the Keplerian angular velocity, in contrast to the previous result.
\end{abstract}

\section{Introduction}

One of the important events of the solar system formation which have not been understood well is the formation of planetesimals. The gravitational instability theories (Safronov, 1969; Goldreich and Ward 1973; Coradini et al., 1981; Sekiya, 1983) are mathematically neat and were preferred previously. It was claimed, however, that the shear induced turbulence might have prevented the dust from settling sufficiently to be gravitationally unstable (Weidenschilling, 1980, 1984; Cuzzi et al., 1993; Weidenschilling and Cuzzi, 1993; Champney et al., 1995; Sekiya, 1998; Dobrovolskis et al., 1999).

In order to elucidate the growth rate of the shear induced instability, we performed linear calculations of the perturbation equations of fluid mechanics in the previous paper (Sekiya and Ishitsu, 2000, hereafter referred to as Paper I) under the following assumptions: (1) The self-gravity is neglected. (2) A mixture of gas and dust is treated as one fluid, which is a good approximation in the case where dust aggregate sizes are small $(\lesssim 1 \mathrm{~cm})$. (3) The solar tidal force, which is the sum of the radial component of the solar gravity and the centrifugal force, is neglected; thus the radial shear $\partial v / \partial r$ is not incorporated in the unperturbed state, and only $z$-component of the solar gravity is taken into account, where $v$ is the rotational velocity of a fluid which is a mixture of gas and dust, $r$ is the distance from the rotation axis, and $z$ is the coordinate perpendicular to the midplane of the solar nebula ( $z=0$ on the midplane). (4) The effects of the Coriolis force are neglected. (5) The effects of the ra-

Copy right (C) The Society of Geomagnetism and Earth, Planetary and Space Sciences (SGEPSS); The Seismological Society of Japan; The Volcanological Society of Japan; The Geodetic Society of Japan; The Japanese Society for Planetary Sciences. dial density and pressure gradients of the unperturbed state are only incorporated in the unperturbed rotation velocity distribution $v_{0}(z)$. (6) Local Cartesian coordinates $(x, y, z)$ are used and we neglect the curvature of a circle with constant value of $r$ and $z$. (7) The unperturbed state has a constant value of the Richardson number in the dust layer. The Richardson number $J$ is a non-dimensional number, which is given by

$$
J=\left[\partial \rho_{0}(z) / \partial z\right] \rho_{0}(z)^{-1} g_{z}\left[\partial v_{0}(z) / \partial z\right]^{-2},
$$

where $\rho_{0}(z)$ is the unperturbed total (gas plus dust) density distribution, $g_{z}=-\Omega_{K}^{2} z$ is the $z$-component of the solar gravity, and $\Omega_{K}$ is the Keplerian angular velocity. A flow is stable as long as $J \geq 0.25$ for all the region (Chandrasekhar, 1961; Howard, 1961).

According to the numerical results of Paper I, the growth rate of the most unstable mode is much less than the Keplerian angular velocity as long as the Richardson number $J$ is larger than 0.1. Thus above assumptions (3) and (4) may be inadequate, since the growth of the most unstable mode require several Keplerian periods and the effects of radial shear and the Coriolis force may not be negligible.

We presumed that the small growth rate of the instability obtained in Paper I is due to special selection of an unperturbed state, i.e. the constant Richardson number solution (Sekiya, 1998), which we adopted only for simplicity. This solution corresponds to a quasi-equilibrium state where the strength of the shear turbulence is just adjusted so that the marginal shear stability is maintained (Sekiya, 1998); thus this density distribution is expected to be achieved asymptotically after the shear induced turbulence had occurred. Before the onset of the shear induced turbulence, there is no reason for the dust density distribution to have been equal 
to the constant Richardson number solution. Thus we use in this paper different unperturbed states, and solve the perturbation equation of the shear stability (Eq. (22) of Paper I), with assumptions (1) to (6) (except (7)) which are mentioned above. In other words, this paper treats the onset of the shear instability in the dust layer which had been laminar, and dust had settled towards the midplane till then. After the onset of the instability, dust density distribution would achieved the quasi-equilibrium density distribution (Sekiya, 1998). Planetesimals are expected to have been formed by mutual sticking of dust aggregates in the nebula with the shear-induced turbulence.

\section{Models and Numerical Results}

As the dust particles settle toward the midplane, they stick each other and aggregates of them grow. In a laminar nebula, the dust settling velocity is proportional to the dust radius (as long as the Epstein law is appropriate) and the distance from the midplane $z$ (equation (5) of Nakagawa et al. (1981)). Thus, dust aggregates grow faster in regions with larger $|z|$, since the principal relative velocity of dust aggregates is induced by difference of settling velocities of dust aggregates with different radii (Weidenschilling, 1980; Nakagawa et al., 1981). As dust aggregates grow, their settling velocities increase. Thus, dust aggregates accumulate in a certain region with an intermediate value of $|z|$ (see 1000 yrs and 1300 yrs density distribution in figure 2 of Nakagawa et al. (1981)). This state is unstable for the Rayleigh-Taylor instability, and the dust density distribution is considered to be adjusted as to be constant in the dust layer (Watanabe and Yamada, 2000). Thus, a dust density distribution has a tendency to be constant in the dust layer. Considering this conjecture, we here adopt a model which has a constant density distribution around the midplane with sinusoidal transition region:

$$
\rho_{d 0}(z)= \begin{cases}\rho_{d 0}(0) & \text { for }|z| \leq z_{d}-2 h \\ \rho_{d 0}(0)\{1- & \left.\left.\sin \left[\pi\left(z-z_{d}+h\right) / 2 h\right)\right]\right\} / 2 \\ & \text { for } z_{d}-2 h<|z|<z_{d} \\ 0 & \text { for } z_{d} \leq|z|\end{cases}
$$

where $\rho_{d 0}(0)$ is the dust density on the midplane, $z_{d}$ is the half-thickness of the dust layer, and $h$ is the half-thickness of the transition zones, where the dust density varies from $\rho_{d 0}(0)$ to 0 sinusoidally.

We consider two representative cases for $h$ : (1) $h / z_{d}=$ 0.5 where dust distribution is given by a sinusoidal curve (no constant density regions), and (2) $h / z_{d}=0.1$ where dust density is constant for $80 \%$ of the dust layer, and density abruptly change in thin transition regions sinusoidally (Fig. 1). As for values of $\rho_{d 0}(0)$, we consider two representative cases where $(\mathrm{A}) \rho_{d 0}(0)=\rho_{g}$ and $(\mathrm{B}) \rho_{d 0}(0)=100 \rho_{g}$, where $\rho_{g}$ is the gas density, which is assumed to be constant in the dust layer (this assumption is valid, since we suppose a thin dust layer). Thus, there are four combinations as listed in Table 1.

We here restrict ourselves to the terrestrial orbit, and the half-thickness of the dust layer is given by

$$
z_{d}=\left[\Sigma_{d} / 2 \rho_{d 0}(0)\right]+h,
$$



Fig. 1. The distribution of the unperturbed dust density $\rho_{d 0}$ given by Eq. (2), in the cases where $h / z_{d}=0.5$ (sinusoidal for $0 \leq|z| / z_{d} \leq 1$ ) and $h / z_{d}=0.1$ (constant for $0 \leq|z| / z_{d} \leq 0.8$, and sinusoidal for $\left.0.8 \leq|z| / z_{d} \leq 1\right)$. Only the region with $z \geq 0$ is depicted.

Table 1. Representative parameters used for a comparative study.

\begin{tabular}{ccccc}
\hline model & $1 \mathrm{~A}$ & $1 \mathrm{~B}$ & $2 \mathrm{~A}$ & $2 \mathrm{~B}$ \\
\hline$h / z_{d}$ & 0.5 & 0.5 & 0.1 & 0.1 \\
$\rho_{d 0}(0) / \rho_{g}$ & 1.0 & 100 & 1.0 & 100 \\
\hline
\end{tabular}

where the dust column density is given by

$$
\Sigma_{d}=7.1 f_{d}\left[\mathrm{~g} / \mathrm{cm}^{2}\right]
$$

We assume $f_{d}=1$ in most of calculations (this is the case of Hayashi's solar nebula model at 1AU (Hayashi, 1981; Hayashi et al., 1985)), except for Fig. 10. Note that the unperturbed total density is given by $\rho_{0}(z)=\rho_{d 0}(z)+\rho_{g}$.

Figures 2 and 3 show the distributions of the Richardson number $J$ (see Eq. (1)) for models (1A) and (1B), and (2A) and (2B), respectively. The Richardson number $J$ is smaller than the critical value 0.25 for most of regions in models (1A) and (1B) (Fig. 2), and for thin layers with sinusoidal density distributions in models (2A) and (2B) (Fig. 3). On the other hand, the Richardson number $J$ is larger than the critical value for regions with constant density distributions in models (2A) and (2B) (Fig. 3).

The growth rate of the instability is calculated under the unperturbed state described above by solving the linear perturbation equation (see equation (22) of Paper I):

$$
\begin{gathered}
\frac{d^{2} w_{1}}{d z^{2}}+\frac{1}{\rho_{0}} \frac{d \rho_{0}}{d z} \frac{d w_{1}}{d z}-\left(k^{2}+\frac{1}{\bar{v}} \frac{d^{2} v_{0}}{d z^{2}}\right. \\
\left.+\frac{1}{\rho_{0}} \frac{d \rho_{0}}{d z} \frac{1}{\bar{v}} \frac{d v_{0}}{d z}+\frac{\Omega_{K}^{2} z}{\bar{v}^{2}} \frac{1}{\rho_{0}} \frac{d \rho_{0}}{d z}\right) w_{1}=0,
\end{gathered}
$$

where $w_{1}$ is the component of the perturbed velocity vertical to the midplane, $\rho_{0}\left(=\rho_{g}+\rho_{d 0}\right)$ is the total unperturbed density, $k$ is the azimuthal wave number (we assume the radial 


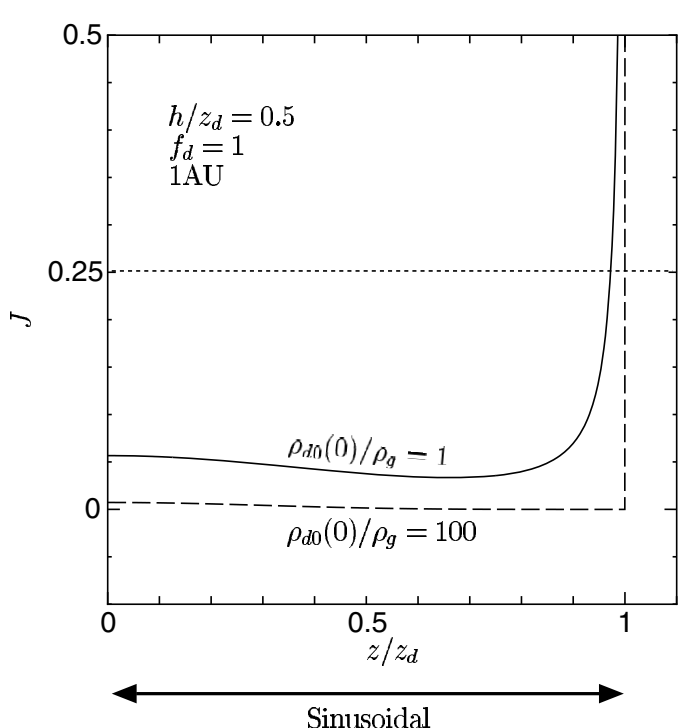

Fig. 2. The distributions of the Richardson number $J$ given by Eq. (1), for models (1A) (solid curve), and (1B) (dashed curve). The flow is stable as long as $J \geq 0.25$ for all the region (Chandrasekhar, 1961; Howard, 1961). The dotted line shows the critical value $J=0.25$.



Fig. 3. Same as Fig. 2, but for model (2A) (solid curve) and (2B) (dashed curve).

wave number to be zero as Paper I), $\bar{v}=v_{0}-(\omega / k)$, and $\omega$ is the complex angular frequency (the perturbation grows if $\omega_{i}$ (the imaginary part of $\omega$ ) is positive). Condition at the boundary between the dust and gas layers is given by (see equation (28) of Paper I)

$$
\frac{d w_{1}}{d z}+\left(k-\frac{1}{\bar{v}} \frac{d v_{0}}{d z}\right) w_{1}=0 \text { at } z=z_{d},
$$

and we assume anti-symmetry with respect to the midplane as Paper I:

$$
w_{1}=0 \text { at } z=0 .
$$

We solved Eq. (5) with boundary conditions, Eqs. (6) and

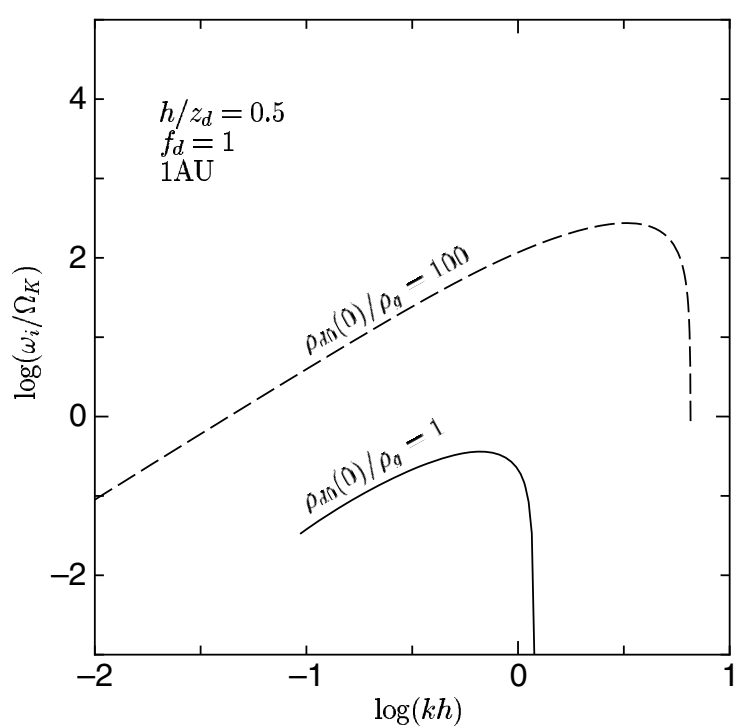

Fig. 4. The growth rates of the shear instability $\omega_{i}$ normalized by the Keplerian angular frequency $\Omega_{K}$ as functions of the wave number $k$ normalized by the half-thickness of the transition region $h$, for models (1A) (solid curve), and (1B) (dashed curve).

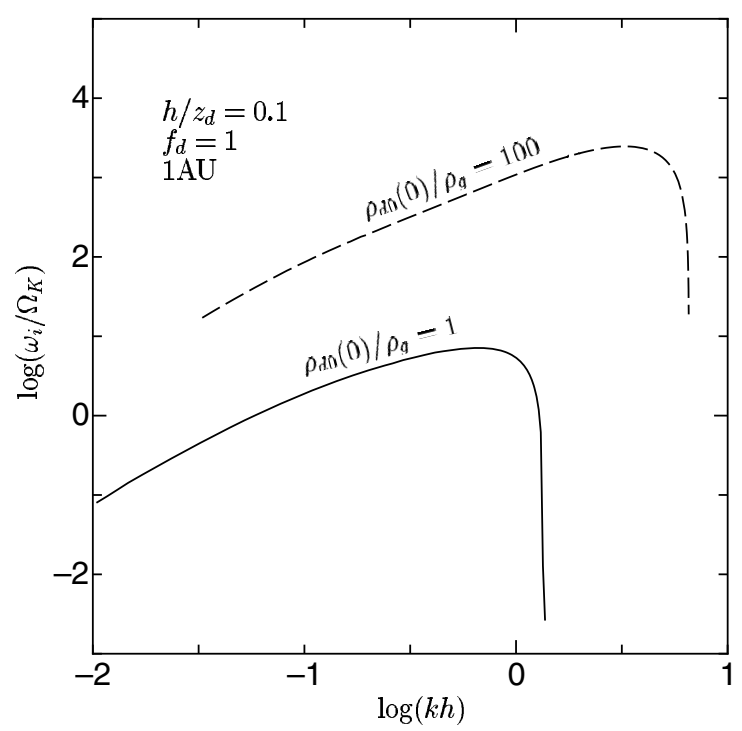

Fig. 5. Same as Fig. 4, but for models (2A) (solid curve) and (2B) (dashed curve).

(7), and obtained the eigenvalue $\omega$.

Figures 4 and 5 show the growth rates of the shear instability as functions of the wave number $k$ in cases (1A) and (1B), and (2A) and (2B), respectively. As seen from these figures, there is a peak growth rate at a finite wave number in each case. This feature is similar to the results of Paper I, where the reason why a peak growth rate exists for a finite wave number is explained in detail.

Figure 6 shows the peak growth rates as functions of $h / z_{d}$ for $\rho_{d 0}(0) / \rho_{g}=1.0$ and 100 . The peak growth rate increases as $h / z_{d}$ decreases, as seen from these figures. Thus a dust layer with abrupt density change is very unstable.

Figure 7 shows the peak growth rates as functions of $\rho_{d 0}(0) / \rho_{g}$ for $h / z_{d}=0.5$ and 0.1 . As seen from this figure, 


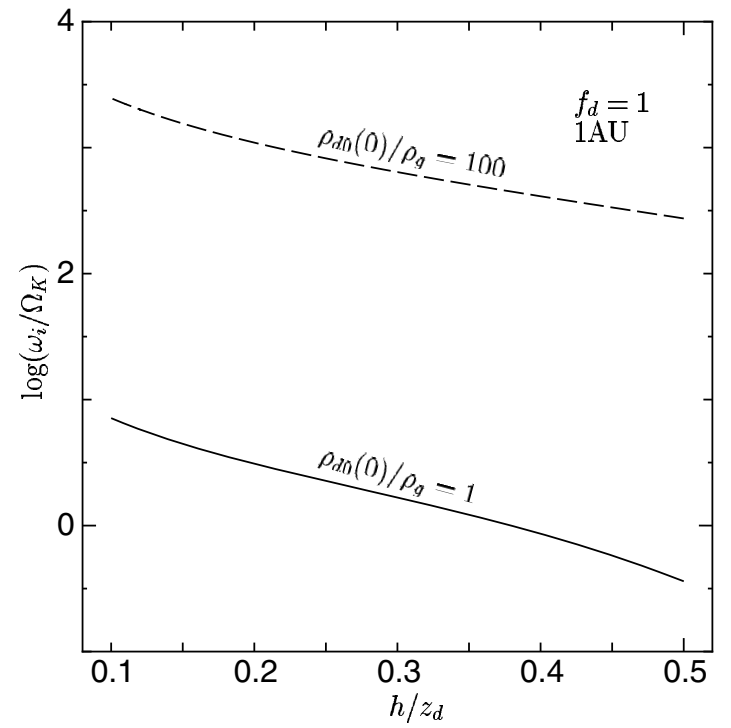

Fig. 6. The peak growth rates of the shear instability $\omega_{i}$ normalized by the Keplerian angular frequency $\Omega_{K}$ as functions of the half-thickness of the transition region $h$ normalized by the half-thickness of the dust layer $z_{d}$, for $\rho_{d 0}(0) / \rho_{g}=1.0$ (solid curve) and 100 (dashed curve).

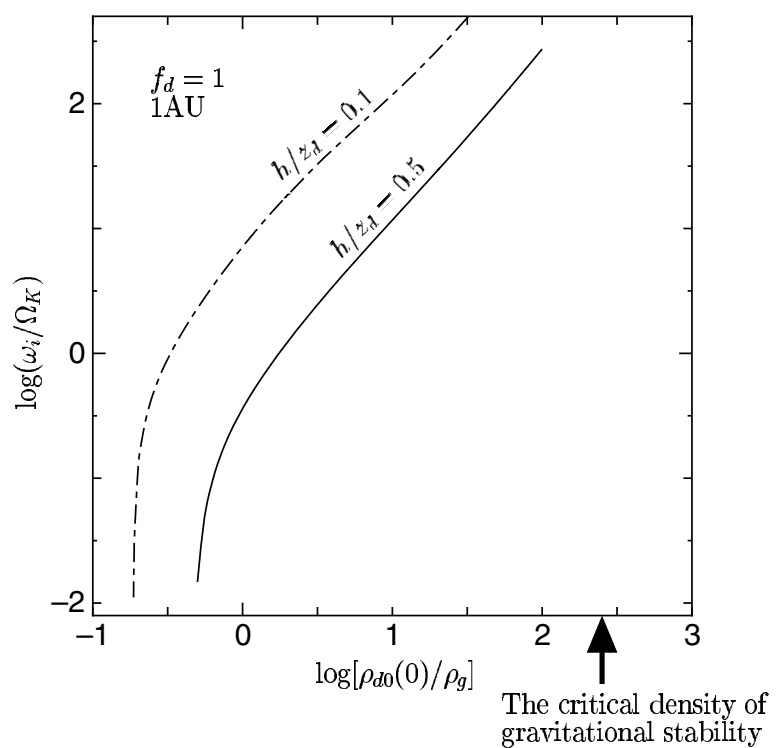

Fig. 7. The peak growth rates of the shear instability as functions of the dust to gas mass ratio on the midplane $\rho_{d 0}(0) / \rho_{g}$, for $h / z_{d}=0.5$ (solid curve) and 0.1 (dash-dotted curve). The critical density of the gravitational stability is shown by the arrow.

the peak growth rate increases as $\rho_{d 0}(0) / \rho_{g}$ increases. The dust layer becomes more unstable as the dust settling proceeds. The gravitational instability of the dust layer could occur if $\rho_{d 0}(0) / \rho_{g}$ (the midplane dust density divided by the gas density) exceed 260, in the case of the Hayashi's nebula model at $1 \mathrm{AU}$ from the Sun (see equations (1) and (13) of Sekiya (1998)). It is seen that the growth rate of the shear instability becomes much larger than the Keplerian angular velocity before the dust layer becomes gravitationally unstable (our assumption to neglect the effects of radial shear and the Coriolis force is appropriate in this case). On the

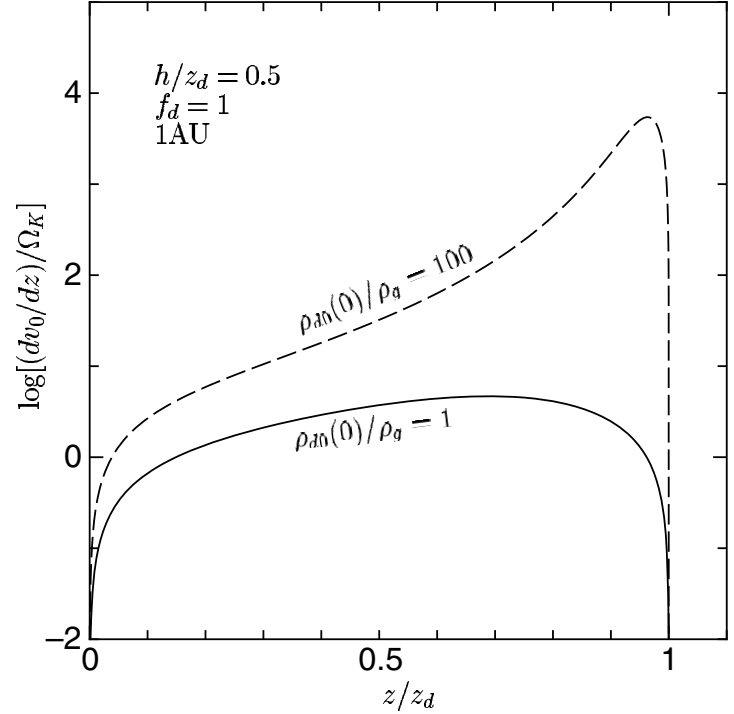

Fig. 8. The shear rates of the unperturbed states normalized by the Keplerian angular velocity, $\left(d v_{0} / d z\right) / \Omega_{K}$, for models (1A) (solid curve) and (1B) (dashed curve).

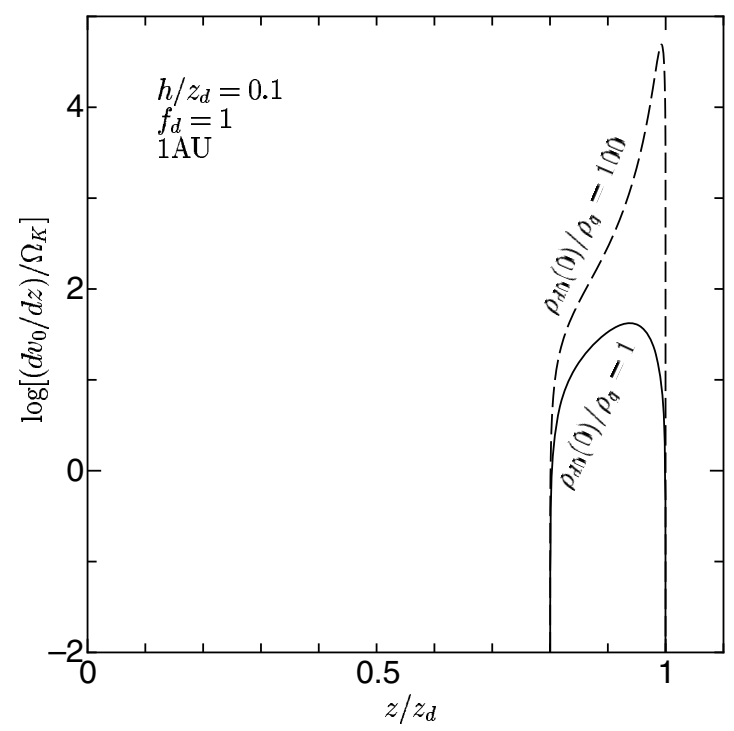

Fig. 9. Same as Fig. 8, but for models (2A) (solid curve) and (2B) (dashed curve).

other hand, time scale of the dust settling is much longer than the Keplerian period, since we assume that dust aggregates are small $(\lesssim 1 \mathrm{~cm})$. Thus the dust layer would be stirred by turbulence induced by the shear instability. The dust density distribution is considered to have converged to a certain state where dust settling and the turbulent diffusion balanced each other (Cuzzi et al., 1993; Champney et al., 1995; Sekiya, 1998; Dobrovolskis et al., 1999).

Figures 8 and 9 show the shear rates of the unperturbed state normalized by the Keplerian angular velocity, $\left(d v_{0} / d z\right) / \Omega_{K}$ for models (1A) and (1B), and (2A) and (2B), respectively. It is seen that as $h / z_{d}$ decreases and $\rho_{d 0}(0) / \rho_{g}$ increases, the peak value of the shear rate increases. Thus, it is seen that the growth rate of the shear instability increases 


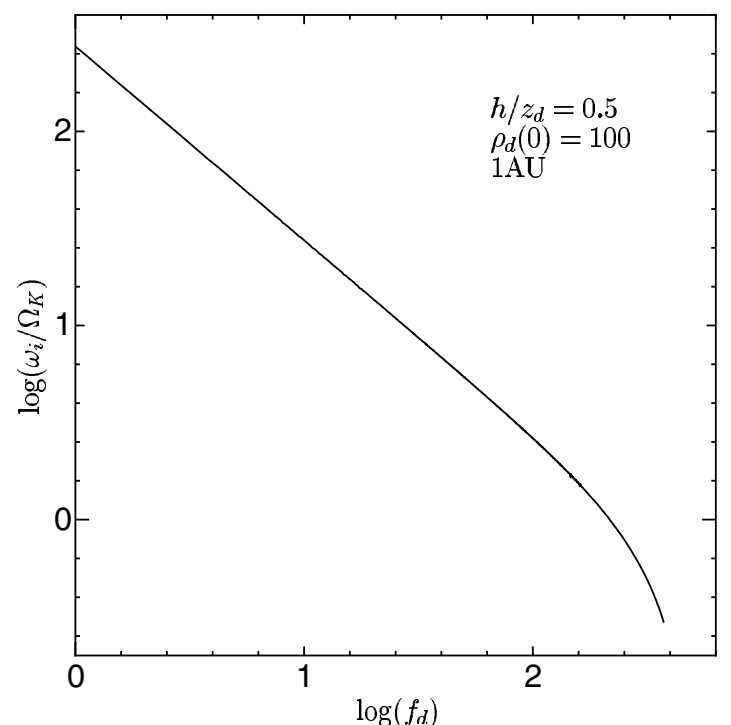

Fig. 10. The peak growth rates of the shear instability as a function of $f_{d}$ (see Eq. (4)) for $h / z_{d}=0.5$ and $\rho_{d}(0)=100$.

as the peak value of the shear rate increases.

Figure 10 shows the peak growth rate as a function of $f_{d}$ (see Eq. (4)). As $f_{d}$ increases, the peak growth rate decreases, which is understood as follows. As $f_{d}$ increases with $\rho_{d}(0)=$ constant, the dust scale height increases, and the shear rate $d v_{0} / d z$ decreases. Thus the shear instability is depressed.

The results of this paper show that the growth rate of the instability is much larger than the Keplerian angular frequency as long as $f_{d} \sim 1$, in contrast to the result of Paper I. The large growth rates obtained in this paper are considered to result from very small values of the Richardson number $J$ in the transition region $z_{d}-2 h<z<z_{d}$ (see Figs. 2 and 3). In other words, the kinetic energy supplied by the shear $d v_{0} / d z$ is converted to the kinetic energy of the perturbed flow if $J \approx 0$; on the other hand, a considerable part of the kinetic energy supplied by the shear is lost through the work done by $z$-component of the solar gravity $\Omega_{K}^{2} z$ in the case of Paper I where $J \gtrsim 0.1$, and the growth of the perturbed flow is suppressed.

\section{Conclusion}

We calculated the growth rate of the shear instability which is induced by density gradient vertical to the midplane of the solar nebula. In this paper, we adopted two types of dust density distributions: (1) a sinusoidal density distribution, and (2) a constant density around the midplane with sinusoidal transition regions, instead of the dust density distribution under the constant Richardson number adopted in the previous paper (Paper I). The results show that the growth rate is much larger than the Keplerian angular velocity, as long as the dust density on the midplane is much larger than the gas density, in contrast to the previous result (Paper I). The dust layer is considered to evolve to a turbulent state, and the dust density distribution would converge to a certain state where dust settling and the turbulent diffusion balanced each other (Cuzzi et al., 1993; Champney et al., 1995; Sekiya, 1998; Dobrovolskis et al., 1999). The dust density in this state is much lower than the critical density of the gravitational instability. Thus planetesimals are considered to have been formed by mutual sticking of dust aggregates. Further elaborate analyses of the shear-induced turbulence are needed in order to elucidate the formation process of planetesimals in more details. For example, in the quasi-equilibrium state obtained by Sekiya (1998), the growth rate of the shear instability is much smaller than the Keplerian angular velocity as calculated in Paper I. In this state, however, the radial shear and the Coriolis force may played important roles. In subsequent papers, we plan to elucidate these problems.

Acknowledgments. We thank anonymous referees for constructive comments. Numerical calculations were performed partly on VX/4R at the Astronomical Data Analysis Center of the National Astronomical Observatory, Japan.

\section{References}

Champney, J. M., A. R. Dobrovolskis, and J. N. Cuzzi, A numerical turbulence model for multiphase flows in the protoplanetary nebula, Phys. Fluids, 7, 1703-1711, 1995.

Chandrasekhar, S., Hydrodynamic and Hydromagnetic Stability, Oxford Univ. Press, Oxford, 1961.

Coradini, A., C. Federico, and G. Magni, Formation of planetesimals in an evolving protoplanetary disk, Astron. Astrophys, 98, 173-185, 1981.

Cuzzi, J. N., A. R. Dobrovolskis, and J. M. Champney, Particle-gas dynamics in the midplane of a protoplanetary nebula, Icarus, 106, 102-134, 1993.

Dobrovolskis, A. R., J. S. Dacles-Mariani, and J. N. Cuzzi, Production and damping of turbulence by particles in the solar nebula, J. Geophys. Res., 104(E21), 30805-30815, 1999.

Goldreich, P. and W. R. Ward, The formation of planetesimals, Astrophys. J., 183, 1051-1061, 1973.

Hayashi, C., Structure of the solar nebula, growth and decay of magnetic fields and effects of magnetic and turbulent viscosities on the nebula, Prog. Theor. Phys. Suppl., 70, 35-53, 1981.

Hayashi, C., K. Nakazawa, and Y. Nakagawa, Formation of the solar system, in Protostars and Planets II, edited by D. C. Black and M. S. Matthews, pp. 1100-1153, Univ. of Arizona Press, Tucson, 1985.

Howard, L. N., Note on a paper of John W. Miles, J. Fluid Mech., 10, 509$512,1961$.

Nakagawa, Y., K. Nakazawa, and C. Hayashi, Growth and sedimentation of dust grains in the primordial solar nebula, Icarus, 45, 517-528, 1981.

Safronov, V. S., Evolution of the Protoplanetary Cloud and Formation of the Earth and the Planets, Nauka, Moscow, NASA Tech. Trans. F-677, 1969.

Sekiya, M., Gravitational instabilities in a dust-gas layer and formation of planetesimals in the solar nebula, Prog. Theor. Phys., 69, 1116-1130, 1983.

Sekiya, M., Quasi-equilibrium density distributions of small dust aggregations in the solar nebula, Icarus, 133, 298-309, 1998.

Sekiya, M. and N. Ishitsu, Shear instabilities in the dust layer of the solar nebula I. The linear analysis of a non-gravitating one-fluid model without the Coriolis and the solar tidal forces, Earth Planets Space, 52, 517-526, 2000.

Watanabe, S. and T. Yamada, Numerical simulations of dust-gas 2-phase flows in the solar nebula, Eos Trans. Am. Geoph. Union Suppl., 81, No. 22, WP99, 2000.

Weidenschilling, S. J., Dust to planetesimals: Settling and coagulation in the solar nebula, Icarus, 44, 172-189, 1980.

Weidenschilling, S. J., Evolution of grains in a turbulent solar nebula, Icarus, 60, 553-567, 1984.

Weidenschilling, S. J. and J. N. Cuzzi, Formation of planetesimals in the solar nebula, in Protostars and Planets III, edited by E. H. Levy and J. I. Lunine, pp. 1031-1060, Univ. of Arizona Press, Tucson, 1993.

M. Sekiya (e-mail: sekiya@geo.kyushu-u.ac.jp) and N. Ishitsu 